\title{
A propeller superficial transverse cervical artery perforator flap for defect coverage of the submental area: a case report
}

Jong Yun Choi,

Jeong Hwa Seo,

Won Jin Cha,

Bommie Florence Seo, Sung-No Jung

Department of Plastic and

Reconstructive Surgery, Uijeongbu St. Mary's Hospital, College of Medicine,

The Catholic University of Korea, Seoul, Korea
Reconstruction of submental defects is a challenge that needs to be approached carefully, since many important anatomical structures are located in this small space. Both aesthetic and functional outcomes should be considered during reconstruction. In this report, we describe a case where a superficial branch of the transverse cervical artery (STCA) perforator propeller flap was applied for coverage of the submental area. An 85-year-old woman presented with a 3-cm ovoid mass on her submental area. We covered the large submental defect with a STCA rotational flap in a $180^{\circ}$ propeller pattern. The flap survived well without any complications at 1 year of followup. A STCA propeller flap is a useful surgical option in reconstruction for defect coverage of the submental area.

Abbreviation: STCA, superficial branch of the transverse cervical artery

Keywords: Case reports / Clavicle / Neck / Neoplasms / Perforator flap / Rotation

\section{INTRODUCTION}

Skin and soft tissue defects in the submental area can require various reconstructive methods. A number of reconstructive options have been reported, including local flap, distant flap, and free flap. However, there are many challenges in submental reconstruction due to the unique characteristics of this area.

The neck and shoulder play a major role in the motion of the head, which is one of the most important parts of the body. For this reason, satisfactory results in terms both functional and cosmetic aspects are critical in reconstruction of the submental area $[1,2]$.

The skin of the neck and shoulder area is somewhat tight, making available donor sites limited compared to other parts of

\footnotetext{
Correspondence: Sung-No Jung

Department of Plastic and Reconstructive Surgery, Uijeongbu St. Mary's Hospital, College of Medicine, The Catholic University of Korea, 271 Cheonbo-ro, Uijeongbu 11765 , Korea

E-mail: jsn7190@catholic.ac.kr

Received November 11, 2021 / Revised November 24, 2021 / Accepted December 18, 2021
}

the body. For this reason, free flap has been the preferred option for head and neck reconstructions in recent years [3,4].

However, in the case of elderly patients with multiple underlying diseases, free flap can be problematic with regard to existing morbidities, so other surgical options should be considered in such cases.

In this report, we describe a successful case where we applied a propeller rotational flap based on a transverse cervical artery perforator for coverage of a large submental defect after a wide neoplasm excision in the head and neck area.

\section{CASE REPORT}

An 85-year-old woman presented to our hospital with a large mass in the submental area, which she first noticed 6 months prior and had increased in size over time. On physical examination, a 3-cm-sized ovoid, dense soft tissue mass was observed in the submental area (Fig. 1). Magnetic resonance imaging was performed to confirm the size, location and nature of the mass. 


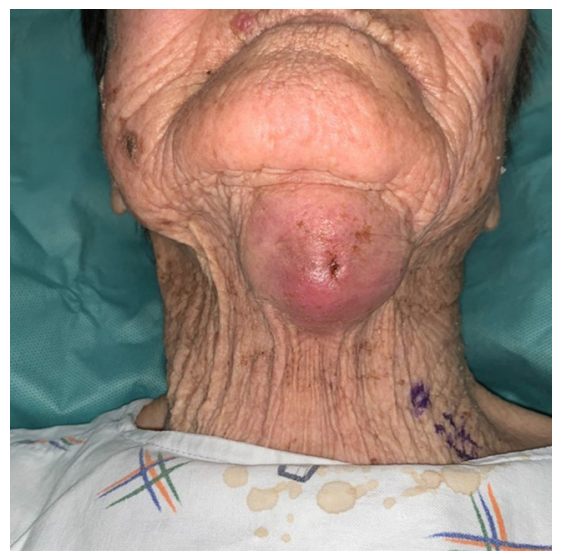

Fig. 1. An 85-year-old woman with a large mass in the submental area.

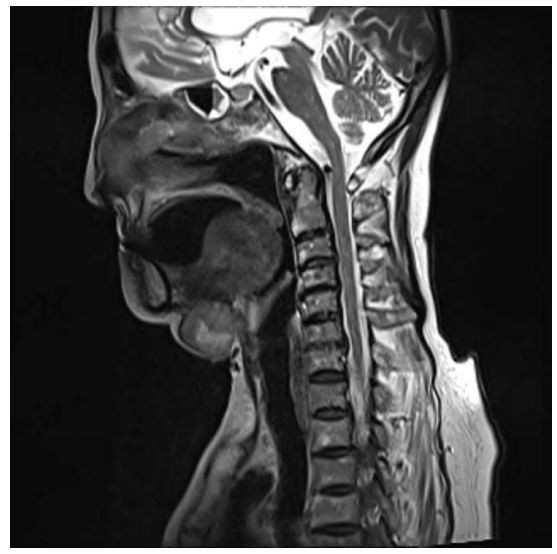

Fig. 2. T2-weighted magnetic resonance imaging revealing a 3-cmsized, ovoid, heterogeneously enhanced mass in the submental area.

A 3-cm-sized ovoid, heterogeneously enhanced mass in the submental area was observed on both T2- and T1-weighted images with slight enhancement, located lateral to the left sternocleidomastoid muscle; several lymph nodes less than $1 \mathrm{~cm}$ in size also showed high signal intensity. Fine needle aspiration biopsy was performed of both the mass and lymph nodes (Fig. 2). The final diagnosis was well-differentiated squamous cell carcinoma.

Wide excision and en bloc resection of the mass and lymph node dissection were performed by an otorhinolaryngologic surgeon, and a $12 \times 6-\mathrm{cm}$-sized submental defect remained after excision. The defect was impossible to close directly; coverage with a flap was necessary for maintaining range of motion in this area.

We designed a superficial branch of the transverse cervical artery (STCA) flap for reconstruction of the defect based on the angiosome. Then, we elevated a flap with abundant fat tissue and identified the perforator from the STCA using a handheld

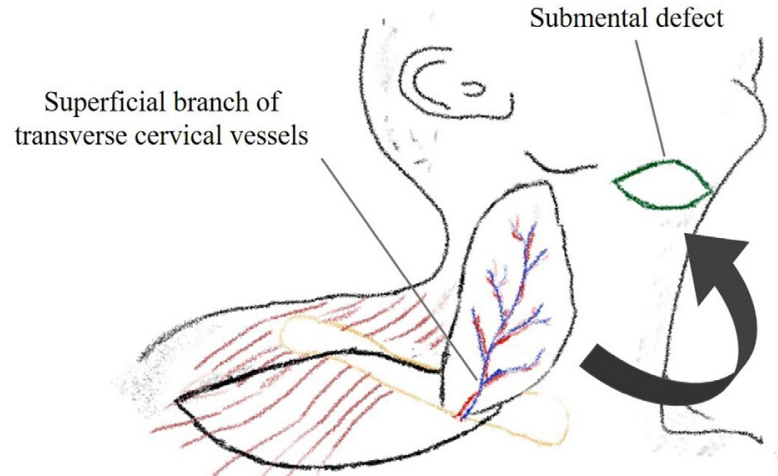

Fig. 3. Scheme of the surgical technique. A superficial transverse cervical artery perforator flap is elevated and passed through a skin tunnel to cover the submental defect.
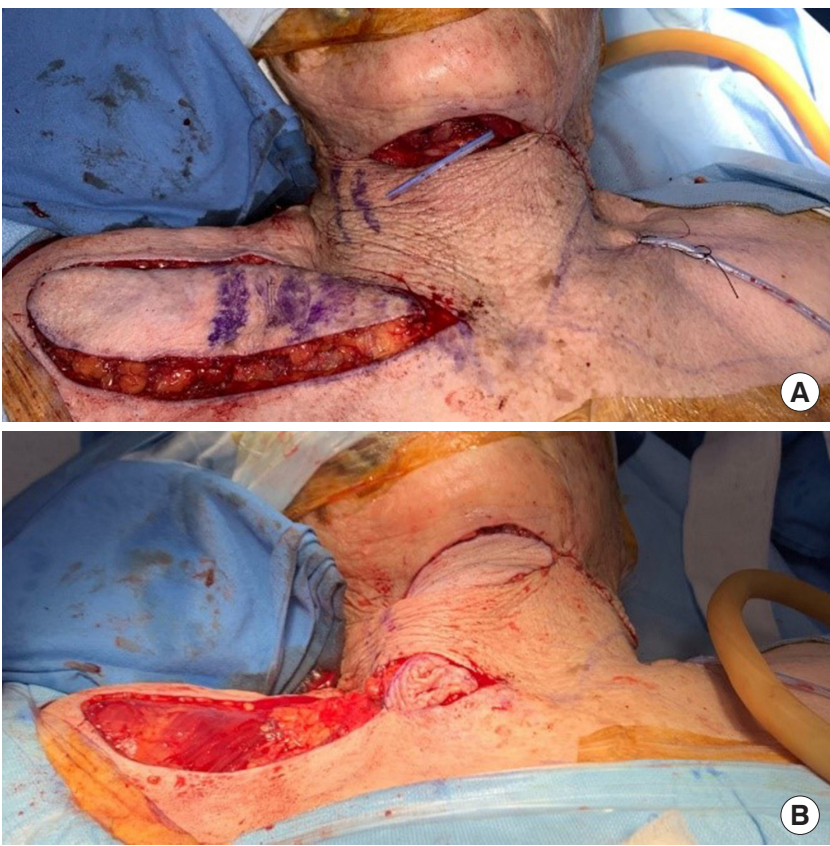

Fig. 4. Intraoperative photographs showing the superficial transverse cervical artery perforator flap rotated $180^{\circ}$ in a propeller fashion to cover the large defect. (A) Flap elevation. (B) Flap insetting.

Doppler device. After identification of the perforator, we designed a pivot point right above the donor site on the right clavicle. The one dominant perforator originated from just below the right clavicle. The flap was elevated by dissecting deep fascia with abundant fat tissue (Fig. 3). After elevating the flap, we performed subcutaneous dissection to the skin flap, tunneling through the submental defect area. This allowed us to safely take the rotated flap through the tunnel below the skin. The middle part of the flap that passed through the skin tunnel had been de-epithelialized. The flap was rotated below the tunnel in a propeller pattern and the defect in the submental area was 


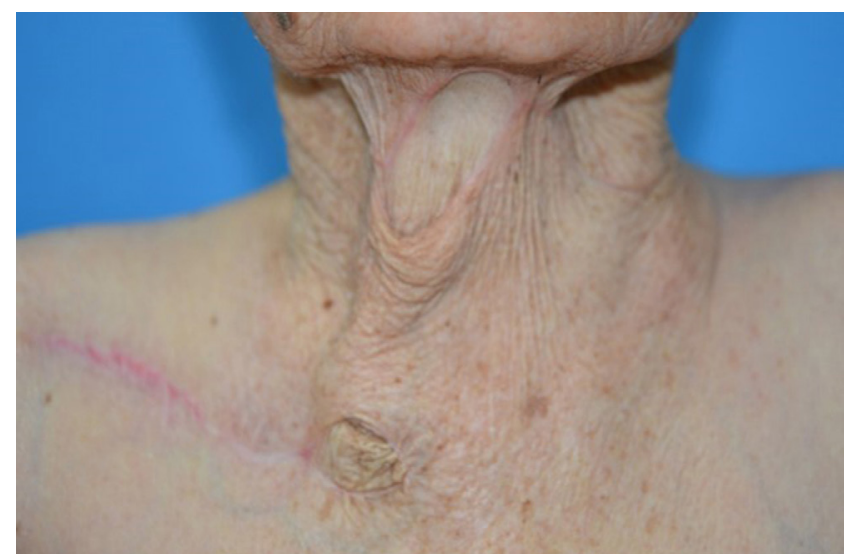

Fig. 5. One-year follow-up photograph.

covered without tension. Then the donor site was immediately sutured with minimal tension (Fig. 4). The flap remained viable without color change throughout admission (12 days postoperative). The patient was stable during the 1 year follow-up period without any complications, and was satisfied with both cosmetic and functional outcomes, reporting no limitations of cervical motion (Fig. 5).

\section{DISCUSSION}

Reconstruction options in the submental area should consider aesthetic, functional, and anatomical aspects [5]. The head and neck area is a very visible part of the body, so matching the color and texture of the skin is important to cosmetic outcomes. Free flaps can yield good functional results, but bulkiness and mismatched skin color remain unsolved issue. There have been several recent reports on rotational flaps as a more useful option than free flaps. The supraclavicular skin is an attractive donor site for neck reconstruction because its features are very similar to the skin of the neck. Morris et al. [6] first introduced the transverse cervical neurovascular free flap. In a cadaver study, an average of four perforators originated from the transverse cervical artery [7]. In another retrospective cadaver study of 103 supraclavicular flaps, $90 \%$ of supraclavicular arteries came from the middle third of the clavicle; thus, the clavicle can be a useful landmark for identifying the angiosome. However, vascular anomalies of the supraclavicular artery exist [8]. Some anatomical studies on how to improve the reliability of supraclavicular flaps have argued that anatomical knowledge of local perforasomes and vascular systems is very important because the supraclavicular flap is mostly based on relatively small and superficial perforators [9-11].

Recently, Kneser et al. [12] introduced a case of reconstruction with a transverse cervical artery perforator propeller flap in a 61-year-old male patient. In this case, use of the anterior trapezius muscle based on a single perforator allowed propeller rotation and primary closure of the donor site from the posterior neck. This is different from our case in that our flap was based on the STCA just below the clavicle, not on the trapezius muscle.

Ismail and Elshobaky [13] reported on the length of supraclavicular flaps for defect coverage. They recommended that the harvested flap should not exceed $22 \mathrm{~cm}$ in length, and flaps should be expanded before harvesting. They suggested that this guideline is helpful for increasing the flap surface and decreasing donor site morbidity.

A propeller flap was defined by Hyakusoku et al. [14] as an island flap that reaches the recipient site through axial rotation. In our case, the pivot point was elevated together with a perforator at the donor site and the harvested flap was rotated in a propeller pattern. Furthermore, a $12 \times 6-\mathrm{cm}$-sized neck defect was able to be covered by tunneling below the subfascial layer with de-epithelialization of the flap.

Our method has several advantages over conventional methods. First, by shortening operation time, the flap survival rate can be increased and the risk of complications can be reduced. Second, the donor site can be sutured immediately and two-dimensional reconstruction of the defect site is possible. Third, compared to free flaps, these propeller flaps are thinner and have a better color match, and require a relatively short learning curve for junior surgeons.

A STCA perforator propeller flap is considered an excellent option for covering extensive submental defects without affecting neck motion. In this case, we achieved both satisfactory functional and esthetic outcomes using such a flap.

\section{NOTES}

\section{Conflict of interest}

Sung-No Jung is an editorial board member of the journal but was not involved in the peer reviewer selection, evaluation, or decision process of this article. No other potential conflicts of interest relevant to this article were reported.

\section{Ethical approval}

The study was approved by the Institutional Review Board of Catholic Medical Center (IRB No. UC21ZASA0143).

\section{Patient consent}

The patient provided written informed consent for the publication and the use of her images. 


\section{ORCID}

Jong Yun Choi

https://orcid.org/0000-0002-1164-4499

Jeong Hwa Seo

https://orcid.org/0000-0002-0911-4747

Won Jin Cha

https://orcid.org/0000-0003-0761-4251

Bommie Florence Seo https://orcid.org/0000-0002-6907-5962

Sung-No Jung https://orcid.org/0000-0002-0419-4717

\section{Author contribution}

Conceptualization: Sung-No Jung. Data curation: Jong Yun Choi. Formal analysis: Jeong Hwa Seo, Won Jin Cha. Methodology: Jong Yun Choi. Project administration: Jong Yun Choi. Visualization: Jeong Hwa Seo, Won Jin Cha. Writing - original draft: Jong Yun Choi. Writing - review \& editing: Jeong Hwa Seo, Sung-No Jung. Investigation: Jeong Hwa Seo. Supervision: Bommie Florence Seo, Sung-No Jung.

\section{REFERENCES}

1. Pallua N, Magnus Noah E. The tunneled supraclavicular island flap: an optimized technique for head and neck reconstruction. Plast Reconstr Surg 2000;105:842-51.

2. Ma X, Zheng Y, Xia W, Fan X, Li Y, Guo S, et al. An anatomical study with clinical application of one branch of the supraclavicular artery. Clin Anat 2009;22:215-20.

3. Suh JM, Chung CH, Chang YJ. Head and neck reconstruction using free flaps: a 30-year medical record review. Arch Craniofac Surg 2021;22:38-44.

4. Kim HS, Chung CH, Chang YJ. Free-flap reconstruction in recurrent head and neck cancer: a retrospective review of 124 cases. Arch Craniofac Surg 2020;21:27-34.

5. Baik B, Park S, Ji S, Kim S. Giant basal cell carcinoma of the left lateral neck. Arch Craniofac Surg 2021;22:173-6.

6. Morris RL, Dillman D, McCabe JS, Holdredge T, Carson L, Given KS. The transverse cervical neurovascular free flap. Ann Plast Surg 1983;10:90-8.

7. Cordova A, Pirrello R, D’Arpa S, Jeschke J, Brenner E, Moschella F. Vascular anatomy of the supraclavicular area revisited: feasibility of the free supraclavicular perforator flap. Plast Reconstr Surg 2008;122:1399-409.

8. Vinh VQ, Van Anh T, Ogawa R, Hyakusoku H. Anatomical and clinical studies of the supraclavicular flap: analysis of 103 flaps used to reconstruct neck scar contractures. Plast Reconstr Surg 2009;123:1471-80.

9. Ross RJ, Baillieu CE, Shayan R, Leung M, Ashton MW. The anatomical basis for improving the reliability of the supraclavicular flap. J Plast Reconstr Aesthet Surg 2014;67:198-204.

10. Pignatti M, Pinto V, Docherty Skogh AC, Giorgini FA, Cipriani $\mathrm{R}$, De Santis G, et al. How to design and harvest a propeller flap. Semin Plast Surg 2020;34:152-60.

11. Pignatti M, Ogawa R, Mateev M, Georgescu AV, Balakrishnan $\mathrm{G}$, Ono S, et al. Our definition of propeller flaps and their classification. Semin Plast Surg 2020;34:139-44.

12. Kneser U, Beier JP, Dragu A, Arkudas A, Horch RE. Transverse cervical artery perforator propeller flap for reconstruction of supraclavicular defects. J Plast Reconstr Aesthet Surg 2011;64: 952-4.

13. Ismail H, Elshobaky A. Supraclavicular artery perforator flap in management of post-burn neck reconstruction: clinical experience. Ann Burns Fire Disasters 2016;29:209-14.

14. Hyakusoku H, Yamamoto T, Fumiiri M. The propeller flap method. Br J Plast Surg 1991;44:53-4. 